\title{
Differences in Circulating Carnitine Status of Preterm Infants Fed Fortified Human Milk or Preterm Infant Formula
}

\author{
*Judit Bene, *Katalin Komlósi, *Bela I. Melegh, 'Tamás Decsi, \\ ${ }^{\ddagger}$ Berthold Koletzko, and ${ }^{\ddagger}$ Ulrike Sauerwald
}

\begin{abstract}
Objective: The aim of the study was to compare plasma carnitine profiles in fortified human milk (HM)-fed preterm infants or formula-fed preterm infants.

Methods: Plasma acylcarnitine concentrations were determined in 20 formula-fed and $18 \mathrm{HM}$-fed preterm infants (birth weights between 1000 and $2200 \mathrm{~g}$ ) by isotope dilution ESI MS/MS technique on study days 0,14 , and 28.

Results: Concentrations of free carnitine (FC) and different acylcarnitines did not change during the 4 weeks of the study in infants fed HM. In contrast, in infants fed formula FC increased markedly (day 0: 29.989 [16.646] $\mu \mathrm{mol} / \mathrm{L}$, median [interquartile range], day 14: 43.972 [8.455], $P<0.05)$ along with increases of short-chain esters (C2 day 0: 5.300 [3.272], day 14: 6.773 [2.127], $P<0.05$; C3 day 0: 0.070 [0.059], day 14: 0.110 [0.069], $P<0.05)$. In contrast, some medium-chain (C8:1, C12) and long-chain esters (C14, C16) decreased significantly in infant formula by day 14, whereas FC and $\mathrm{C} 2$ and $\mathrm{C} 3$ esters increased further by day 28 (FC: 47.672 [14.753], C2: 7.430 [4.688], C3: 0.107 [0.047]).

Conclusions: The altered carnitine ester profile likely reflects active involvement of the carnitine molecule in the buffering, metabolism, and elimination of nonphysiological acyl moieties.
\end{abstract}

Key Words: carnitine ester profile, human milk, mass spectrometry, premature infant, preterm infant formula

(JPGN 2013;57: 673-676)
B efore birth, amino acids and glucose are the main substrates for fetal metabolism. The human placenta is also permeable to small amounts of triglycerides, fatty acids, glycerol, and keto acids. A few hours after delivery, the neonate must switch from glucose use to oxidation of fat, which becomes the major source of energy $(1,2)$.

Carnitine, which is present in the body in free and esterified form (acylcarnitines [ACs]), plays an indispensible role in the energy metabolism by controlling the influx of long-chain fatty acids into the mitochondrial matrix where they undergo $\beta$-oxidation $(3,4)$. The fetus probably obtains exogenous carnitine via placental transport. Carnitine is stored in fetal tissues in increasing amounts during the third trimester of pregnancy, and tissue stores at birth are directly related to gestational age $(5-10)$; thus, preterm infants are born with limited carnitine reserves (11). Because endogenous synthesis of carnitine is limited in the neonate, preterm infants cannot achieve carnitine homeostasis without its exogenous supply (12).

The amount of circulating carnitine as well as carnitine esters in preterm infants depends on the nutritional status. Different ways of infant feeding may influence the use of carnitine through either fat or the amino acid content of the nutrients. We investigated carnitine levels longitudinally by using mass spectrometry (MS) in preterm neonates fed human milk (HM) or infant formula (IF) during the period of 28 days to assess the effect of way of feeding on carnitine homeostasis.

\section{METHODS}

\section{Subjects and Sample Collection}

We studied samples of 38 preterm infants (20 formula-fed

Received January 24, 2013; accepted June 8, 2013.
From the *Departments of Medical Genetics, the †Paediatrics, University of Pécs, Pécs, Hungary, and the $\ddagger$ Kinderklinik and Kinderpoliklinik, Dr von Hauner Children's Hospital, University of Munich Medical Centre, Munich, Germany.

Address correspondence and reprint requests to Dr Judit Bene, Department of Medical Genetics, University of Pécs, Szigeti 12, H-7624 Pécs, Hungary (e-mail: bene.judit@pte.hu).

This work was supported by OTKA 73430, ETT 210-07/2009 and ÁOKKA 34039-6/2009, Deutsche Forschungsgemeinschaft, Bonn, Germany (KO 912/5-1 and 5-2), and Nestlé Nutrition, Vevey, Switzerland and Frankfurt/Main, Germany. Further support was provided by the Commission of the European Communities, within the 7th Framework Programme, NUTRIMENTHE, FP7-212652. The content of this article does not necessarily reflect the views of the Commission and in no way anticipates the future policy in this area.

The authors report no conflicts of interest.

Copyright $(\mathcal{C}) 2013$ by European Society for Pediatric Gastroenterology, Hepatology, and Nutrition and North American Society for Pediatric Gastroenterology, Hepatology, and Nutrition

DOI: $10.1097 / \mathrm{MPG} .0 \mathrm{~b} 013 \mathrm{e} 31829 \mathrm{fad} 06$ and 18 human milk-fed) with birth weights between 1000 and $2200 \mathrm{~g}$ who had participated in a previously reported feeding trial (13). Briefly, participating infants were recruited from the neonatal units of 5 German hospitals and included in the study within the first 14 days of life (postnatal age was $13 \pm 1$ days for formula-fed, and $12 \pm 3$ days for human milk-fed infants) after full enteral feeding was established $\left(>80 \mathrm{~mL} \cdot \mathrm{kg}^{-1} \mathrm{day}^{-1}\right)$ and at least $80 \%$ of the caloric intake was derived from either human milk or preterm formula (based on Beba preterm infant formula, Nestlé Nutrition, Frankfurt, Germany), which contained $1.7 \mathrm{mg}$ carnitine and $4.16 \mathrm{~g}$ fat in $100 \mathrm{~mL}$ standard solution (13). Human milk was fortified with FM 85 (Nestlé Nutrition) that contained no carnitine. Before study entry, infants were fed according to hospital routine.

Infants with severe malformations, apparent genetic, gastrointestinal or metabolic disorders, artificial ventilation or oxygen supply $>30 \%$ at the time of enrollment, and administration of 
parenteral fat emulsion $\left(>1 \mathrm{~g} \cdot \mathrm{kg}^{-1} \cdot \mathrm{day}^{-1}\right.$ triglycerides for $>7$ days) before study entry were excluded from the study.

Venous blood samples were collected in ethylenediaminetetraacetic acid tubes from all infants on study days 0,14 , and 28. Plasma was separated by centrifugation ( 5 minutes, $1000 \mathrm{~g}$, room temperature) and stored at $-20^{\circ} \mathrm{C}$ until analysis.

Informed consent was obtained from all of the parents before the study. During the entire study period, the guidelines and regulations approved by the University of Munich Medical Centre were followed and the study was carried out in accordance with the Helsinki Declaration of 1975, as revised in 2000.

\section{MS Analysis}

Free carnitine (FC) and all the ACs were determined in butylester forms using isotope dilution MS method. Flow injection analysis was applied for the individual measurements, which were carried out in a Micromass Quattro Ultima ESI (electrospray ionization) triple-quadrupole mass spectrometer coupled with a Waters 2795 HPLC (Milford, MA) system for sample introduction.
For sample preparation, $10 \mu \mathrm{L}$ of plasma was used and then a previously described procedure was followed (14). During the ESI MS/MS analysis, FC and ACs were measured by positive precursor ion scan of $\mathrm{m} / \mathrm{z} 85$, with a scan range of $\mathrm{m} / \mathrm{z} 200$ to 550 . The applied capillary voltage, cone voltage, and collision energy were $2.54 \mathrm{kV}, 55 \mathrm{~V}$, and $26 \mathrm{eV}$, respectively. Our mass spectrometry facility is registered participant in the International Newborn Screening Quality Assurance Program organized by the Centers for Disease Control and Prevention (http://www2.cdc.gov/nceh/ NewbornScreening).

\section{Statistical Analysis}

All statistical analyses were performed with SPSS version 20 (SPSS Inc, Chicago, IL). Variables are presented as median and interquartile range. The effect of diet on plasma carnitine ester concentration on days 0,14 , and 28 was studied with MannWhitney $U$ tests. Changes in carnitine ester concentrations over time within groups (day 0 to day 14 to day 28) were analyzed using the Friedman test. If changes were found to be significant, Wilcoxon

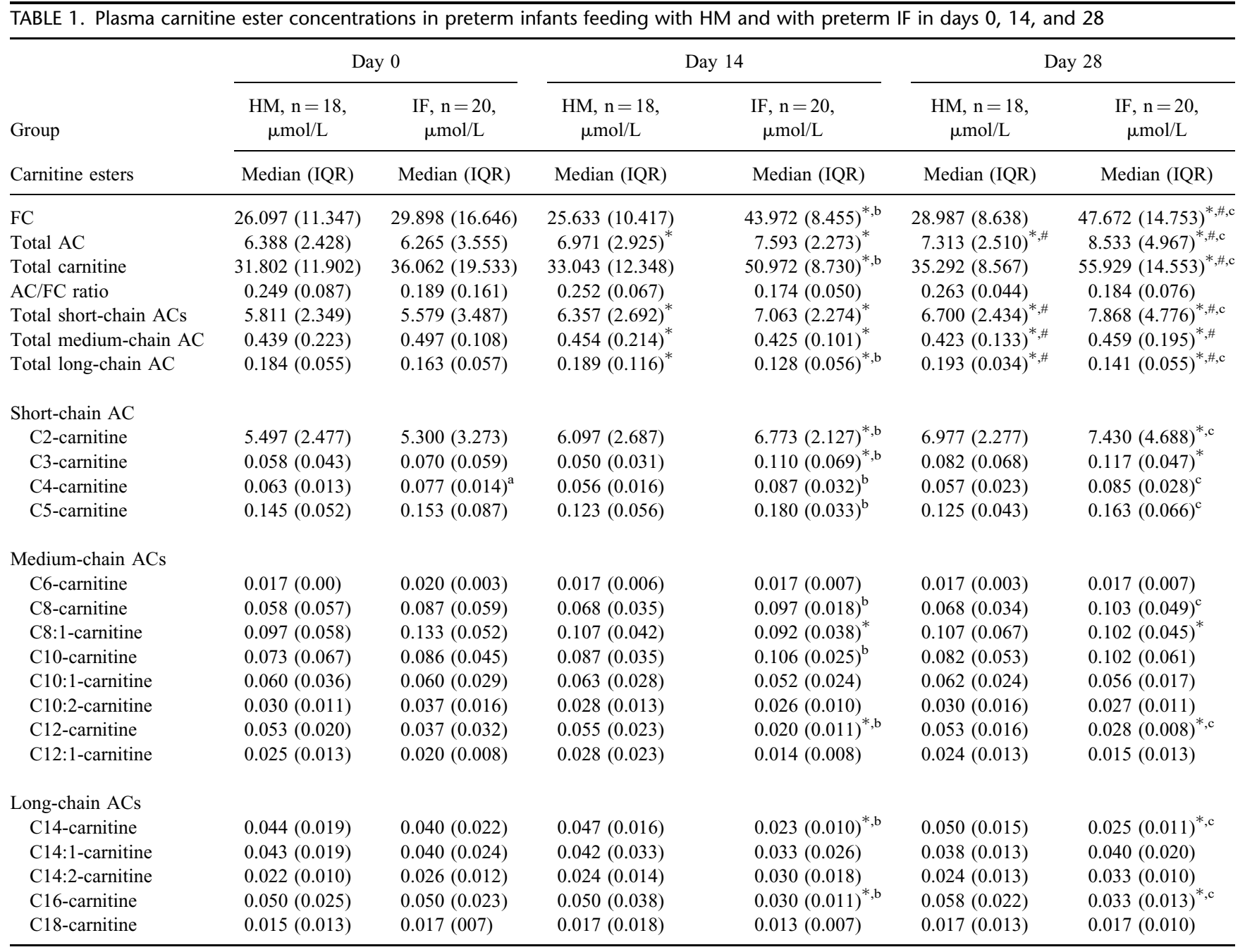

Different superscript a, b, c indicate significant differences between groups on the same day $(P<0.05)($ Mann-Whitney $U$ test $)$. AC $=$ acylcarnitines; $\mathrm{FC}=$ free carnitine; $\mathrm{IQR}=$ interquartile range.

* Significantly different from study day $0(P<0.05)$ (Wilcoxon test).

${ }^{\#}$ Significantly different from study day $14(P<0.05)$ (Wilcoxon test). 
signed rank tests were performed. The level of statistical significance was set at $P<0.05$ for all tests.

\section{RESULTS}

The plasma levels of FC and different ACs are summarized in Table 1. Virtually no statistically significant difference was observed in the levels of either FC or individual esters in the human milk fed (HM) infants when comparing the results obtained at days 0, 14 and 28. Contrarily, the amounts of total short-chain (SCAC), total medium-chain, total long-chain (LCAC), and finally total ACs (TAC) displayed alterations.

In the preterm formula-fed infants (IF) we did not detect any differences in $\mathrm{FC}$ and $\mathrm{AC}$ according to the subgroups receiving different docosahexaenoic acid intakes; therefore, results for all IF infants are grouped together. At day 14, a marked increase in FC along with significantly elevated $\mathrm{C} 2$ and $\mathrm{C} 3$ esters, total short-chain esters, and total esters was found, as compared with the results of day 0 . Furthermore, the amount of some medium-chain (C8:1, C12) and long-chain esters (C14, C16) decreased significantly. The levels of FC and C2 and C3 esters increased further to day 28, which resulted in the significant elevation of the total short-chain and finally the total AC and total carnitine (TC) levels at day 28 compared to the results of day 0 . Simultaneously, the values of C8:1, $\mathrm{C} 12, \mathrm{C} 14$, and C16 remained decreased at day 28.

Comparison of the results of HM-fed and IF-fed groups revealed significant differences in the concentrations of FC and different ACs between the 2 groups during the study period. At baseline (day 0), there was no significant difference in the levels of FC and individual ACs between the 2 study groups; however, 14 days later, marked changes were observed. The levels of FC (Fig. 1) and all of the short-chain esters were found to be markedly higher in the IF group than in the HM group (by $20 \%-70 \%$ of the day 14 values of $\mathrm{HM}$ ); moreover, the amounts of C8 and C10 were also found to be significantly higher (by $39 \%$ and $16 \%$ of the day 14 value of HM, respectively). In addition, the levels of C12, C14, and $\mathrm{C} 16$ esters were considerably lower in the IF group than in the $\mathrm{HM}$ group (by $50 \%$ of the day 14 values of $\mathrm{HM}$ ). There were no statistically significant differences in the levels of other ACs between the 2 study groups.

At day 28, there was no substantial further change in the carnitine ester pattern between the 2 study groups when comparing the results with those of day 14 . The changes in the levels of FC and different ACs resulted in a significantly higher levels of total SCACs, total ACs (Fig. 1). and lower levels of total LCACs in the IF group compared with the HM group. In spite of the lower levels of LCAC, the levels of TC are still higher in the IF group than in the HM group (Fig. 1).

\section{DISCUSSION}

Carnitine has been considered as a conditionally essential nutrient for human adults, children, neonates, and, especially, premature infants. Neonates have a reduced ability to synthesize carnitine, and premature neonates have reduced tissue stores because they lack the major portion of placental carnitine transfer, which takes places during the third trimester; hence, they largely rely on exogenous carnitine after birth (11). During the past few decades, a large number of publications established physiological functions of carnitine and revealed its relevance in normal and pathological conditions (15-19); however, the methodology used earlier was based on the radioenzymatic determination of carnitine. Using alkaline hydrolysis, only the determination of F and TC levels and calculation of the amount of the TC esters was possible without any information of the composition of the acyl groups. The introduction of MS has enabled sensitive measurements of specific carnitine esters. Despite the introduction of this tool into the newborn screening program for inborn errors of metabolism (20), studies on carnitine ester evolution in neonates are scarce $(21,22)$.

In this study, we examined markers of carnitine metabolism longitudinally during 4 weeks in preterm infants HM or IF. During the 1-month follow-up, no substantial changes were observed in the HM fed group. Our present data suggest that feeding HM leads to development of a steady state condition in the plasma carnitine levels regardless of the weight gain and increasing human milk intake during this period of time.

Although the infant formula used in this study contains about twice as much FC as found in human milk (approximately $40-50$ $\mu \mathrm{mol} / \mathrm{L}(23)$ ), remarkable alterations were observed in the carnitine ester profile between the 2 study groups. Similarly to Shortland et al (24), we detected a higher amount of FC, TC, and TACs at day 28 in the IF group. In addition, we also detected changes of individual acyl carnitines. Besides the increased levels of SCACs and few medium-chain ACs that may result from the higher medium-chain triglycerides content of the preterm IF, we also observed lower amounts of LCACs in the IF than in the HM group.

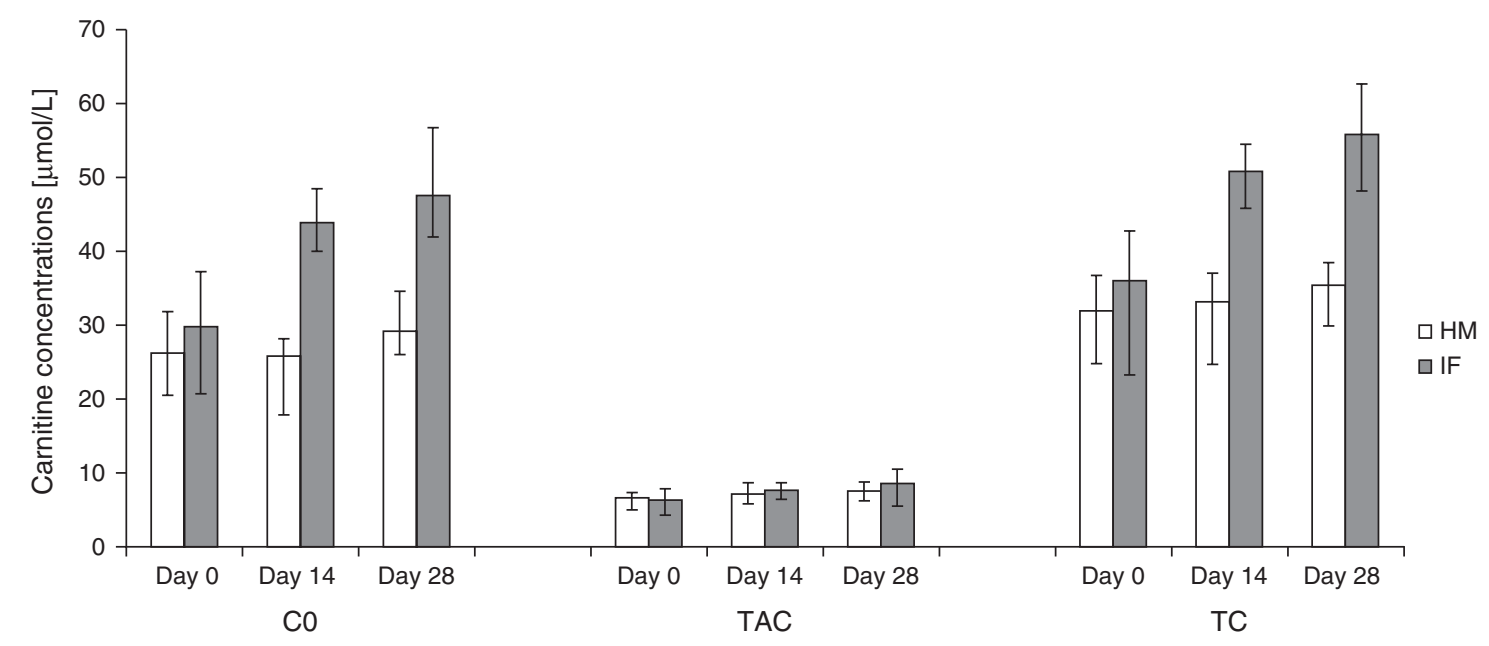

FIGURE 1. Plasma-free carnitine (C0), total acylcarnitine (TAC), and total carnitine (TC) concentrations in preterm infants feeding with human milk (HM) and with preterm infant formula (IF) in days 0,14 , and 28. 
Our results differ from those of Campoy et al (25), who found lower levels of total SCAC, TAC, TC, and higher levels of LCAC in the late neonatal period in full-term infants fed cow's-milk formula compared with breast-fed infants. In contrast, we found higher amounts of total SCAC and lower levels of total LCAC in the IF than in the HM group. A possible reason for these differences may be different methods used to determine carnitine esters. Until now only 1 study was published in which the AC profiles of the preterm neonates were assessed by MS. In this study, Meyburg et al determined longitudinal changes in AC profiles of full-term and preterm neonates with different gestational ages (22); however, the concept of their study differed from ours and hence results are not comparable.

Our results show that formula feeding of preterm infants is metabolically not equivalent to breast-feeding. The protein, amino acid, and fatty acid composition of preterm formula differs from $\mathrm{HM}$; therefore, bulk elimination, metabolism, and detoxification differ. Our data demonstrate the involvement of carnitine metabolism in these processes. Carnitine probably plays an important dynamic role in these complex metabolic events that affects a large number of metabolites. Because the short- and long-term consequences of such metabolic alterations are unknown, it would appear prudent to try to approach the metabolic response of breastfed infants as much as feasible also in infants fed formula.

Acknowledgment: Preterm infant formula for this trial was supplied by Nestlé Nutrition, Frankfurt, Germany.

\section{REFERENCES}

1. Ward PM, Deshpande S. Metabolic adaptation at birth. Semin Fetal Neonatal Med 2005;10:341-50.

2. Bougneres PF, Lemmel C, Ferre P, et al. Ketone body transport in the human neonate and infant. J Clin Invest 1986;77:42-8.

3. Bremer J. Carnitine-metabolism and functions. Physiol Rev 1983; 63:1420-80.

4. Bieber LL. Carnitine. Annu Rev Biochem 1988;57:261-83.

5. Melegh B. Carnitine supplementation in the premature. Biol Neonate 1990;58 (suppl 1):93-106.

6. Melegh B, Kerner J, Sandor A, et al. Effects of oral L-carnitine supplementation in low-birth-weight premature infants maintained on human milk. Biol Neonate 1987;51:185-93.

7. Penn D, Schmidt-Sommerfeld E, Pascu F. Decreased tissue carnitine concentrations in newborn infants receiving total parenteral nutrition. J Pediatr 1981;98:976-8.

8. Penn D, Ludwigs B, Schmidt-Sommerfeld E, et al. Effect of nutrition on tissue carnitine concentrations in infants of different gestational ages. Biol Neonate 1985;47:130-5.
9. Hahn P, Skala JP, Seccombe DW, et al. Carnitine content of blood and amniotic fluid. Pediatr Res 1977;11:878-80.

10. Shenai JP, Borum PR. Tissue carnitine reserves of newborn infants. Pediatr Res 1984;18:679-82.

11. Crill CM, Storm MC, Christensen ML, et al. Carnitine supplementation in premature neonates: effect on plasma and red blood cell total carnitine concentrations, nutrition parameters and morbidity. Clin Nutr 2006; 25:886-96.

12. Whitfield J, Smith T, Sollohub H, et al. Clinical effects of L-carnitine supplementation on apnea and growth in very low birth weight infants. Pediatrics 2003;111:477-82.

13. Sauerwald UC, Fink MM, Demmelmair H, et al. Effect of different levels of docosahexaenoic acid supply on fatty acid status and linoleic and alpha-linolenic acid conversion in preterm infants. $J$ Pediatr Gastroenterol Nutr 2012;54:353-63.

14. Bene J, Komlosi K, Havasi V, et al. Changes of plasma fasting carnitine ester profile in patients with ulcerative colitis. World J Gastroenterol 2006;12:110-3.

15. Talian GC, Komlosi K, Decsi T, et al. Determination of carnitine ester patterns during the second half of pregnancy, at delivery, and in neonatal cord blood by tandem mass spectrometry: complex and dynamic involvement of carnitine in the intermediary metabolism. Pediatr Res 2007;62:88-92.

16. Melegh B, Kerner J, Acsadi G, et al. L-carnitine replacement therapy in chronic valproate treatment. Neuropediatrics 1990;21:40-3.

17. Kispal G, Melegh B, Alkonyi I, et al. Enhanced uptake of carnitine by perfused rat liver following starvation. Biochim Biophys Acta 1987; 896:96-102.

18. Melegh B, Pap M, Morava E, et al. Carnitine-dependent changes of metabolic fuel consumption during long-term treatment with valproic acid. J Pediatr 1994;125:317-21.

19. Vaz FM, Melegh B, Bene J, et al. Analysis of carnitine biosynthesis metabolites in urine by HPLC-electrospray tandem mass spectrometry. Clin Chem 2002;48:826-34.

20. Millington DS, Kodo N, Norwood DL, et al. Tandem mass spectrometry: a new method for acylcarnitine profiling with potential for neonatal screening for inborn errors of metabolism. J Inherit Metab Dis 1990;13:321-4.

21. Meyburg J, Schulze A, Kohlmueller D, et al. Postnatal changes in neonatal acylcarnitine profile. Pediatr Res 2001;49:125-9.

22. Meyburg J, Schulze A, Kohlmueller D, et al. Acylcarnitine profiles of preterm infants over the first four weeks of life. Pediatr Res 2002; 52:720-3.

23. Sandor A, Pecsuvac K, Kerner J, et al. On carnitine content of the human breast milk. Pediatr Res 1982;16:89-91.

24. Shortland GJ, Walter JH, Stroud C, et al. Randomised controlled trial of L-carnitine as a nutritional supplement in preterm infants. Arch Dis Child Fetal Neonatal Ed 1998;78:F185-8.

25. Campoy C, Bayes R, Peinado JM, et al. Evaluation of carnitine nutritional status in full-term newborn infants. Early Hum Dev 1998; 53(Suppl):S149-64. 